\title{
High prevalence of large trematode eggs in schoolchildren in Cambodia
}

Philipp J. Bless ${ }^{\text {a,b }}$, Fabian Schär ${ }^{\text {a,b }}$, Virak Khieu ${ }^{\text {a,b,c }}$, Stefanie Kramme ${ }^{\text {a,b }}$, Sinuon Muth

c, Hanspeter Marti ${ }^{\mathrm{a}, \mathrm{b}}$, Peter Odermatt ${ }^{\mathrm{a}, \mathrm{b}}$

${ }^{a}$ Swiss Tropical and Public Health Institute, P.O. Box, CH-4002 Basel, Switzerland

${ }^{b}$ University of Basel, Basel, Switzerland

${ }^{\mathrm{c}}$ National Center for Parasitology, Entomology and Malaria Control, Ministry of Health, Phnom Penh, Cambodia

Keywords: intestinal helminths, trematodes, Fasciola hepatica, Fasciola gigantica, Southeast Asia, Cambodia

Corresponding author: Peter Odermatt, Department of Epidemiology and Public Health, Swiss Tropical Institute, P.O. Box, CH-4002 Basel, Switzerland. Tel.: +41-6128482 14, Fax: + 41-61-284 81 05; E-mail address: peter.odermatt@unibas.ch (P. Odermatt) 


\begin{abstract}
Large trematode eggs (LTE) resembling Fasciola spp. eggs were reportedly found in the stools of schoolchildren in Kandal province, Cambodia. This study aimed to assess the prevalence of LTE in the stools of children attending the affected school, identify potential risk factors for infection and ascertain the trematode species. We performed a cross-sectional study involving an in-depth questionnaire administered to schoolchildren at the affected school, and examined cattle droppings in the surrounding area and the livers of slaughtered cattle. Three stool samples were examined per child, using KatoKatz and formalin-ether concentration techniques. In addition, blood serum ELISA and coprological PCR was conducted for species clarification. Cattle droppings were examined by cup sedimentation and coprological ELISA. LTE were observed in the stools of 228 schoolchildren (46.5\%). Two blood serum samples from schoolchildren were positive for Fasciola hepatica in a first ELISA but were negative in a confirmation immunofluorescence antibody test. Out of 221 PCR samples, only one tested positive for Fasciola spp. and none for Fasciolopsis buski. The consumption of raw aquatic plants $(\mathrm{OR}=3.3)$ and fermented fish sauce $(\mathrm{OR}=2.1)$ were significantly associated with LTE in the stool. Fasciola spp. flukes were observed in $18.3 \%$ of 191 cattle livers. The prevalence of fascioliasis in cattle droppings was $88.8 \%$. The low prevalence of schoolchildren that tested positive for Fasciola spp. with specific molecular diagnostics and that had no diagnostic evidence of Fasciolopsis buski strongly indicates that the majority of microscopically observed LTE are from Echinostoma spp.. Fasciola sp. transmission from cattle to human is possible and public health services need to be alerted accordingly.
\end{abstract}




\section{Introduction}

In low and middle income countries, intestinal multiparasitism is the rule rather than the exception (McKenzie, 2005; Petney and Andrews, 1998; Steinmann et al., 2010). In Southeast Asia, intestinal helminth and protozoa multiparasitism is well known and has been documented (Lee et al., 2002; Park et al., 2004; Sayasone et al., 2011, 2009; Sinuon et al., 2003). Several endemic species of food-borne trematodes (FBT) have been identified, such as liver flukes (Opisthorchis viverrini, and Fasciola spp.) and intestinal flukes (Fasciolopsis buski, and Echinostoma spp.) (Hien et al., 2001; Keiser and Utzinger, 2005; Quang et al., 2008; Sayasone et al., 2011; Sohn et al., 2011a, 2011b). Helminthic multiparasitism poses a serious challenge for parasitological diagnosis, as eggs from different species may morphologically resemble one another. For example, the size, oval shape and presence of small and inconspicuous operculum of Fasciola spp. eggs are indistinguishable from Fasciolopsis buski and some Echinostoma spp. eggs. The average egg size of all three parasite genera is similar in terms of length and width: Fasciola hepatica 106.5-171.5/63.9-95.4 $\mu \mathrm{m}$, F. gigantica 150.9-182.2/85.1-106.2 $\mu \mathrm{m}$ (Valero et al., 2009), Echinostoma revolutum 97-117/ 61-65 $\mu \mathrm{m}$ (Sohn et al., 2011a); Echinostoma ilocanum 89-99/52-58 $\mu \mathrm{m}$ (Sohn et al., 2011b) and Fasciolopsis buski $\mu \mathrm{m}$. They can be characterised as Fasciola-like eggs or large trematode eggs (LTE). In 2009, LTE were observed in the stools of $27 / 150$ schoolchildren (18.0\%) in the Damrei Chhlang village primary school, Kandal province, Cambodia, during a standard parasitological survey using the Kato-Katz technique (Khieu et al., 2013). Given the high prevalence of Fasciola spp. infection among cattle in Southern Cambodia (Tum et al., 
2007), LTE could have been attributable to Fasciola spp. infection. However, other trematode species could also have been responsible.

The aim of this study was to assess the prevalence of LTE in the stools of schoolchildren, identify risk factors for infection and ascertain the trematode species, all with a focus on Fasciola spp.. We performed a cross-sectional study among the schoolchildren, examined cattle droppings in and around Damrei Chhlang village and inspected cattle livers in a local slaughterhouse.

\section{Materials and methods}

\subsection{Ethical considerations}

Ethical clearance for this study was obtained from the Ethics Commission of Basel (EKBB; reference no. 159/11) and from the National Ethics Committee for Health Research (NECHR), Ministry of Health (MOH) in Phnom Penh, Cambodia (reference no. 30/NECHR). Before field work started, the provincial and district health authorities, village and school authorities and parents were informed of the study and, in turn, working permission was granted. Prior to enrolling each participant, written informed consent was obtained from the parents, legal guardian or the participant him/herself if they were of legal age. In cases of illiteracy, the informed consent was signed by fingerprint before the village chief, who signed as a witness.

\subsection{Study design, population and area}

The study was carried out in Central Kandal province, which surrounds Phnom Penh. It is inhabited by 1.2 million people and divided into 11 districts. Between May and June 
2011, a cross-sectional study was carried out among schoolchildren at the Damrei

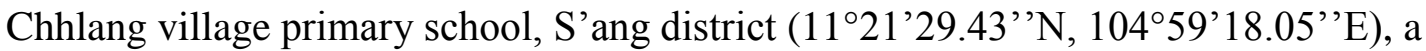
district of 1,831 people.

Another cross-sectional study aimed to estimate the prevalence of fascioliasis in cattle droppings in the villages surrounding the Damrei Chhlang primary school (S'ang district). These villages included Damrei Chhlang and Preaek Khmaer, (11'21'21.70'’N, $104^{\circ} 58^{\prime} 59.33^{\prime \prime}$ E), both located in S'ang Phnum commune; and Preaek Run

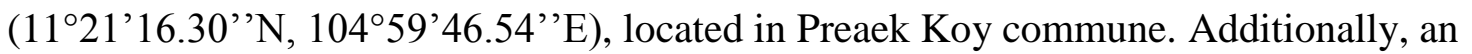
abattoir study of a small facility that slaughtering local cattle was conducted in Preaek Koy commune.

\subsection{Procedures in the field}

On day one, enrolled study participants assembled at the community hall next to the school. A trained interviewer questioned each child about potential risk factors for infection and about experiences of ill-health. Subsequently, a physician assessed participants' general health status and clinical symptoms by using a standardized assessment form. Additionally, a nurse drew a venous blood sample of $5 \mathrm{ml}$ from each child. The blood samples were stored in a cooling box at about $5-10^{\circ} \mathrm{C}$ until they reached the laboratory of the National Center for Parasitology, Entomology and Malaria Control $(\mathrm{CNM})$ in Phnom Penh.

A pre-labeled stool container was given to each child, along with instructions on how to

fill it. Children were asked to collect morning stools to ensure freshness of the stool samples. The following morning, the filled container was collected and a new pre-labeled 
container was provided. Three stool samples were collected from each child over four consecutive days. Each day after collection, containers were directly transported to the laboratory of the CNM for analysis.

The stool containers for cattle droppings were distributed among randomly selected cattle owners, each of whom collected one fresh stool sample from every one of their cattle. Containers were collected in the morning, the day after distribution, and directly transported to the laboratory of the CNM.

The butcher at the study abattoir examined the livers of slaughtered cattle on a continuous basis for meat quality assurance. For study purposes, he recorded in a diary the total number of slaughtered cattle and of Fasciola spp. infected livers.

\subsection{Laboratory procedures}

For each of the three stool samples per child, one Kato-Katz thick smear (Katz et al., 1972) was prepared directly after the samples arrived in the laboratory. After a half an hour clearance time, the smears were examined under a light microscope (magnification 400x) for intestinal helminth eggs. The number of eggs per parasite species was counted and recorded.

From each of the three stool samples per child, approximately 1 gram was preserved in $15 \mathrm{ml}$ sodium acetyl formalin (SAF) fixative for analysis by formalin-ether concentration technique (FECT) (Marti and Escher, 1990). Four weeks after collection, the fixed samples were processed and examined under a light microscope (magnification 1000x) for intestinal helminths and protozoa. 
From the first stool sample per child, approximately 0.5 gram of fresh stool was preserved in $70 \%$ ethanol for later molecular analysis using real-time quantitative Polymer chain reaction (PCR) to diagnose Fasciola sp. (Alasaad et al., 2011) and Fasciolopsis buski infections (unpublished, developed by Dr. med. Stefanie Kramme, Swiss TPH). The preserved samples were sent to the Swiss TPH laboratory in Basel, Switzerland, where molecular analysis was carried-out about two months later. First, from each preserved stool sample, $200 \mu \mathrm{g}$ was washed twice with phosphate buffered saline (PBS) to eliminate the ethanol. Subsequently, DNA was extracted with the QIAamp DNA Stool Mini Kit (QIAGEN, USA). The extracted DNA was frozen at $-20^{\circ} \mathrm{C}$ until PCR was performed, one week later. On 0.5 gram of the first stool samples from each child, a commercially available $F$. hepatica coproantigen enzyme-linked immunosorbent assay (ELISA test) (Bio-X, Belgium) was performed at the CNM laboratory.

Upon arrival, blood samples were stored at room temperature to clot. Subsequently, the blood samples were centrifuged and the serum was frozen and stored at $-20^{\circ} \mathrm{C}$ at the CNM laboratory. The frozen samples were sent to the Diagnostic Center of the Swiss TPH, Switzerland, where an ELISA for Fasciola hepatica-antibodies was performed two months after sample collection. Sera samples with a positive result were retested with an immunofluorescence antibody test (IFAT) for confirmation. All sera samples with a positive or critical (close to positive) result were retested with a helminth screening ELISA (unpublished, Dr. Hanspeter Marti, Swiss TPH), which included Fasciola hepatica, Strongyloides stercoralis, Schistosoma spp., Burgia malayi, Wuchereria bancrofti, Echinococcus spp., Toxocara spp. and Trichinella spp.. 
Cattle droppings were stored at $5^{\circ} \mathrm{C}$ in a fridge at the $\mathrm{CNM}$ laboratory until analysis. The next day, cattle droppings were examined by the same $F$. hepatica coproantigen ELISA test (Bio-X, Belgium) that had been used to examine the stool samples from children. Identical procedures were used, only the weight of stool was increased from 0.5 gram to 2 grams. Within 48 hours of collection, cattle droppings were examined a second time by cup sedimentation. Approximately 10 grams of fecal material was mixed with $\mathrm{NaCl} 0.9 \%$ and filtered through a double layer of gauze. The cup was filled with $\mathrm{NaCl} 0.9 \%$ and left to stand for one hour. Three slides of the sediment were examined under a light microscope (magnification 400x) for Fasciola spp. eggs. The left over sediment was centrifuged, mixed with SAF-solution and ether, vigorously shaken, centrifuged again and examined again under the light microscope (magnification 400x).

\subsection{Risk factor assessment}

Potential risk factors for LTE infection were assessed, using a two-part questionnaire.

First, a questionnaire was administered to each schoolchild to collect individual information about basic demography, eating habits and personal hygiene. Second, a household questionnaire was administered to the head of household to obtain demographic information for the head of household and for the participant, as well as information about the participant's recent medical history, eating habits and food consumption, household assets and water and sanitation facilities. The questionnaire provided information about exposure to potential risk factors, general health information, and household. A physical examination of each participant was conducted by qualified CNM staff to collect information about his/her general physical condition, recent 
medical history and other clinical observations. By understanding participants' general health and the household conditions in which they live, the questionnaire and physical exam provided information on exposure to potential risk factors for trematode infection.

\subsection{Data management and analysis}

All data were double entered into Epidata v3.1 (EpiData Association; Odense Denmark), and validated and analysed by Stata v10.1 (Stata Corporation; College Station, TX, USA). The analysis included all schoolchildren with complete individual and household questionnaires, a $5 \mathrm{ml}$ venous blood sample and at least one stool sample examined by Kato-Katz, FECT and PCR analysis.

The gold standard for diagnosing LTE- and co-infections is the compiled results of three Kato-Katz slides and three FECT analyses. Concomitant, a child was defined as LTE positive (outcome) if at least one LTE was observed in either the Kato-Katz and/or FECT examination. The same definition was applied to co-infections. Age groups and sex were tested for differences in LTE or co-infection prevalence with a chi square likelihood ratio test (LRT) or with Fisher's exact test. The diagnostic performance of Kato-Katz vs.

FECT for LTE detection was compared by using the McNemar test or the exact McNemar test. Arithmetic mean of eggs per gram (epg) of feces was calculated for LTE and co-infections. Additionally, the real prevalence of LTE and co-infections was estimated by a prediction model (Marti and Koella, 1993). 
Anthropometric measurements were calculated and stratified for age and sex. Gender distribution, anthropometric measurements and fever were tested for differences with a binomial test (0.5), one sample t-test and one-way ANOVA, respectively.

Each participant's socio-economic status was estimated using a household-based asset approach, in which the study population was stratified into wealth index tertiles: (i) most poor (ii) poor and (iii) least poor. Wealth index tertiles were calculated by principal component analysis (PCA), using the following household assets: cattle/horse cart, farm vehicle with engine, car, motorbike, bicycle, mobile phone, television, radio, electric fan, current generator, direct electricity, animal ownership (chickens, ducks, pigs, cows) and food security (rice field, vegetable garden). The unadjusted first principal component (PC) accounted for $12.5 \%$ of the total variability and its weights were used to calculate the household index score. Finally, asset scores were summarised and assigned to each participant (Houweling et al., 2003).

A univariable logistic regression analysis was performed to associate potential risk factors with the presence of LTE (outcome). Odds ratios (OR), 95\% confidence intervals and p-values were calculated. Variables with a p-value of less than 0.25 in the univariable analysis were selected as possible predictors in the multivariable logistic regression analysis.

The full multivariable model with all preselected variables underwent backward selection and was rated by the use of the Bayesian information criterion (BIC). For the final multivariable model odds ratios (ORs), 95\% confidence intervals and p-values are reported and factors with a p-value of less than $5 \%$ were considered as significant. 
The gold standard diagnosis of fascioliasis in living cattle relied on the combined results from two stool examinations (cup sedimentation and copro-ELISA, Bio-X, Belgium). Cattle were defined as Fasciola spp. positive (outcome) if at least one Fasciola spp. egg was observed in one stool examination. 


\section{Results}

\subsection{Schoolchildren's characteristics}

Of the 257 schoolchildren enrolled, 228 (88.7\%) had complete data records (household questionnaire, individual questionnaire, blood sample and at least one stool sample) and were used in the final analysis (Figure 1). All participating schoolchildren lived in the Damrei Chhlang village.

The children ranged in age from 5 to 18 years, with a median of 11 years and the following age structure: 5-7 years (16.7\%), 8-10 years (29.4\%), 11-13 years (38.2\%) and 14-18 years (15.8\%); 50.9\% were male. Overall mean height was $127.3 \mathrm{~cm}$ (range 95-160 $\mathrm{cm}, \mathrm{SD} 14.7 \mathrm{~cm})$ and males were not significantly taller compared to females $(\mathrm{p}=0.982)$. Mean weight of males and females did not differ significantly $(\mathrm{p}=0.985)$ and overall mean weight was $24.9 \mathrm{~kg}$ (range 10.5-50.0 kg, SD 7.8 kg). Mid Upper Arm Circumference (MUAC) was on average $17.2 \mathrm{~cm}$ (range 10.5-26.0 cm, SD $2.2 \mathrm{~cm}$ ) and did not differ significantly between the sexes $(\mathrm{p}=0.770)$. Mean overall body temperature was $36.1^{\circ} \mathrm{C}$ (range of $34.0-37.8^{\circ} \mathrm{C}, \mathrm{SD} 0.6^{\circ} \mathrm{C}$ ) and did not differ between sexes $(\mathrm{p}=0.847$ ) or age groups $(\mathrm{p}=0.580)$.

\subsection{Infection with large trematode eggs and associated clinical signs}

In almost half of the study participants (46.5\%), LTE were observed in at least one stool examination. There was no significant difference between the sexes $(\mathrm{p}=0.608)$ but infection prevalence differed between age groups $(\mathrm{p}=0.037)$ and was highest among the the group aged 11-13 years (Table 1). 
The prevalence of LTE as determined by the Kato-Katz technique (KK) was $21.9 \%$ and $36.4 \%$ as determined by FECT. There is a significant difference between these two tests $(\mathrm{p}<0.001)$ with a ratio $(\mathrm{KK} / \mathrm{FECT})$ of 0.602 (95\% CI 0.460-0.789). Considering the combined results of three Kato-Katz slides and three FECTs $(n=203)$ as the gold standard for detecting LTE, the cumulative sensitivities for the first, second and third Kato-Katz slide were $30.2 \%, 37.7 \%$ and $47.2 \%$, respectively and for the first, second and third FECT $37.7 \%, 67.0 \%$ and $78.3 \%$, respectively.

Blood samples were tested by serum ELISA and Fasciola-antibodies were detected in two samples. LTE were not observed in these two samples via KK or FECT. Subsequently, the two positive samples were retested by IFAT, which yielded negative test results. Considering the negative IFAT result, the two positive samples and those samples with a critical (close to positive) value after the first serum ELISA were retested with a helminth screening ELISA. No Fasciola-antibodies were detected with this test. Using copro-ELISA, Fasciola-antigens were identified in six participants. Out of these six participants, only two had observable LTE in the stool while one other had a positive serum ELISA result.

Of the 221 human stool samples tested with the Fasciola-specific PCR, one showed a positive result. The $F$. buski-specific PCR was negative for all stool samples. The participant with the positive PCR result had neither LTE in the stool (Kato-Katz, FECT) nor a positive result in serological ELISA.

Study participants were clinically examined and questioned about the presence of clinical signs and symptoms in the two weeks prior to examination, particularly those relating to the suspected parasitic infections. A number of symptoms were reported, including 
abdominal pain (64.5\%), diarrhea (54.0\%), fever (52.6\%), pain in the right upper quadrant (25.0\%), vomiting (21.9\%), itchy skin (15.8\%) nausea (14.9\%) and weight loss (11.8\%). Clinical signs observed by study physicians included generalised rash (2.6\%), pale skin color $(2.2 \%)$, pale subconjunctiva (2.2\%), jaundice (1.8\%) and poor general condition (3.1\%). Only one participant suffered from splenomegaly and none of the participants suffered from ascites or hepatomegaly. Statistical analysis (univariable and multivariable regression analysis) showed that none of the clinical signs and symptoms were associated with LTE infection.

\subsection{Other intestinal parasitic infections}

Overall, $70.2 \%$ of the participants were diagnosed with an intestinal parasitic infection (Table 1). A significant difference in prevalence was observed between age groups $(\mathrm{p}=0.026)$. The highest infection prevalence of more than $75 \%$ was observed in the groups aged 8-10 years and 11-13 years. There was no significant difference between $\operatorname{sexes}(\mathrm{p}=0.644)$.

Besides LTE, another trematode, $O$. viverrini was diagnosed in the study population. The observed prevalence of $O$. viverrini in the study population did not differ between age groups $(\mathrm{p}=0.540)$ or by sex $(\mathrm{p}=0.355)$. Nematode infections presented the biggest diversity, with five different species observed. Most common were hookworms, for which no significant age $(\mathrm{p}=0.214)$ or by sex $(\mathrm{p}=0.382)$ difference was observed. Ascaris lumbricoides had the lowest prevalence, which did not differ significantly between age groups $(\mathrm{p}=0.866)$ or by sex $(\mathrm{p}=0.679)$. Additional nematodes diagnosed were Trichuris trichiura, Enterobius vermicularis and, in one participant, S. stercoralis larvae. 
Hymenolepis nana, Taenia sp., Entamoeba histolytica / dispar / moshkovskii, Gardia

lamblia, Entamoeba. coli and Isospora belli were also detected.

Helminth and intestinal protozoa multiparasitism was common among the participants.

Of the participants, $36.4 \%$ were infected with a single parasite and $33.8 \%$ with more than one species. Participants were infected with two, three and four different parasites species, with a prevalence of $22.8 \%, 8.3 \%$ and $2.6 \%$, respectively.

\subsection{Risk factors for an LTE infection}

In the univariable logistic regression, of the variables describing the demographics of the study participants, only age (11-13 years) was significantly associated with the observation of LTE $(\mathrm{p}=0.012)$ (Table 2). Of the variables describing the anthropometric measurements and fever, none were significantly associated with the observation of LTE. Body temperature, body weight and mid upper arm circumference met the inclusion criteria for the multivariable logistic regression analysis.

Variables pertaining to the participant's eating habits, consumption of raw aquatic plants $(\mathrm{p}=0.029)$ and consumption of fermented fish sauce $(\mathrm{p}=0.032)$, were significantly associated with the observation of LTE. The variables raw fermented fish sauce $(\mathrm{p}=0.210)$, pork meat $(\mathrm{p}=0.227)$ and aquatic plants $(\mathrm{p}=0.238)$ met the inclusion criteria for the multivariable logistic regression analysis. Of the variables describing the participant's hygiene practices, none were significantly associated with the outcome. Only daily bathing in river or pond $(\mathrm{p}=0.178)$ met the inclusion criteria for the multivariable logistic regression analysis. 
In the multivariable logistic regression analysis, the consumption of raw aquatic plants $(\mathrm{OR}=3.3,95 \% \mathrm{CI} 1.3-8.5, \mathrm{p}=0.013)$ and fermented fish sauce $(\mathrm{OR}=2.1,95 \% \mathrm{CI} 1.0-4.4$ $\mathrm{p}=0.046$ ) were significantly associated with the observation of LTE in the stool. The consumption of raw aquatic plants resulted in a three-fold risk increase for an LTE infection, confirming the finding of the univariable logistic regression analysis where the consumption of raw aquatic plants showed the same trend. The consumption of fermented fish sauce doubled the risk for an LTE infection, consistent with the univariable logistic regression analysis, which also showed a significant positive association with LTE infection. Of the remaining variables in the multivariable logistic regression model, i.e. daily bathing in river or pond, itchy skin, generalized rash, general condition, body temperature and mid upper arm circumference, none were significantly associated with the presence of LTE in stool.

\subsection{Fascioliasis study in cattle}

For the study, a sample size of 200 cattle was estimated and 222 cattle were enrolled. The analysis included 205 cattle (Preaek Run (58), Damrei Chhlang (59) and Preaek Khmaer (88)) with complete data records, i.e. 1 stool sample, 1 cup sedimentation and 1 ELISA test. Of the 205 cattle analysed, there were 84 males and 121 females. The overall prevalence of cattle fascioliasis was $88.8 \%$, using the gold standard diagnostics. Among males, the prevalence was $88.1 \%$ and $89.3 \%$ among females.

During the 17-day observation period, a total of 191 cattle were slaughtered and their livers were examined for Fasciola spp. flukes, resulting in the detection of 35 (18.3\%) cattle livers infected with Fasciola spp. 


\section{Discussion}

In this study, we showed that fascioliasis is endemic in the Damrei Chhlang primary school environment. An $18.3 \%$ prevalence rate was observed among the livers of slaughtered cattle, while an average prevalence of $88.8 \%$ was diagnosed from bovine stool from surveyed villages. Our observation is consistent with another study conducted in Kandal province in 2001 and 2002, which found an infection prevalence of $85.2 \%$ (Tum et al., 2007). This high bovine fascioliasis prevalence was predicted by a GIS risk map developed by Tum and colleagues (Tum et al., 2004). Therefore, given the environmental contamination with Fasciola spp. eggs, the proximity of cattle to villagers and the raw food consumption of villagers, zoonotic transmission of the parasite from cattle to human is possible in this area.

In schoolchildren and adolescents, we found a high prevalence $(46.5 \%)$ of LTE in examined stool samples. Two participants tested positive through an ELISA test on blood serum, while one participant's DNA tested positive via a PCR for Fasciola spp.. Our risk factor assessment showed that the consumption of raw or undercooked aquatic plants was a significant risk factor for LTE infection. Furthermore, the veterinary copro-ELISA detected Fasciola-antigens in stool samples from six (of 228) human participants, two of which had observable LTE in the stool.

The prevalence of LTE among study participants was very high and cannot be explained by the Fasciola spp. positive results of the ELISA test on serum or the PCR. In addition, the results of the ELISA test on serum were controversial. The first ELISA detected two Fasciola positive cases. Both cases had a negative confirmatory IFAT. It must be 
mentioned that the antigen used for the ELISA test on serum was crude worm extract of $F$. hepatica. Therefore, the detection of $F$. gigantica-antibodies was only partially or not at all possible. Of the two participants with positive ELISA test results (on serum), one also tested positive by copro-ELISA, but no LTE were found in the stools of either participant. This finding is consistent with findings in Vietnam where eggs in the stool were observed in only 14 of 285 fascioliasis cases (Hien et al., 2001). Finally, only two participants had a Fasciola spp. positive PCR result. Given these results, we conclude that it is very unlikely that the observed LTE are Fasciola spp. Eggs, but rather belong to Fasciolopsis or Echinostoma trematode species.

Generally, the presence of Echinostoma spp. in Southeast Asia is well known (Chai et al., 2009). Different Echinostoma species have been reported in Cambodia's neighboring countries: Lao PDR (Chai et al., 2012; Sohn et al., 2013), Thailand (Radomyos et al., 1998; Tungtrongchitr et al., 2007; Waree et al., 2001) and Vietnam (Chai et al., 2011). At the beginning of the 2000s, the first Echinostoma spp. infections were reported in Cambodian schoolchildren, with prevalences ranging from $4.8 \%$ to $15.6 \%$ (Lee et al., 2002; Park et al., 2004).

In 2011, Sohn and colleagues demonstrated the presence of Echinostoma revolutum in Pursat province and Echinostoma ilocanum in Oddar Meanchey province, Cambodia, by noting the distinct morphological characteristics of adult flukes recovered from human patients (Sohn et al., 2011a, 2011b). Their published pictures of E. ilocanum eggs observed during Kato-Katz analysis were strikingly similar to the LTE observed during our Kato-Katz analyses (Figure 3), suggesting that the LTE observed in our study were 
most likely due to Echinostoma spp. infection. We did not systematically collect LTE measurement data and hence, cannot compare it with Sohn et al.'s measurement data. Surprisingly, the risk factors identified in our study were not consistent with those for Echinostoma infection. In fact, we found that the consumption of raw aquatic plants and fermented fish sauce were significant risk factors for LTE eggs in stool. The consumption of raw aquatic plants is a well-known way of acquiring a Fasciola spp. or $F$. buski infection. However, humans acquire Echinostoma spp. infections in Cambodia by consuming uncooked snails, clams and freshwater fish (Sohn et al., 2011a, 2011b). In our study, the consumption of raw or undercooked fish was not reported by any participant. Cooked fish consumption was reported by nearly all study participants and it was not significantly associated with an increased risk of LTE in stool in the univariable analysis. It is evident that some of these participants were consuming raw or undercooked fish, as Opisthorchis viverrini infection was detected in a considerable number of participants. This in-depth investigation documents once more the high degree of intestinal helminthic infection, multiparasitism and diversity in a semi-rural setting in Cambodia. Almost three-quarters $(70.2 \%)$ of the study participants harbored a helminth infection. Multiparasitism was observed in one-third (33.8\%) of the participants. Even higher rates were observed in rural Laos (Sayasone et al., 2011, 2009). Besides LTE infection, we diagnosed seven additional helminth species. At this point, it must be noted that Opisthorchis viverrini infection diagnosed through Kato-Katz analysis were also confirmed in FECT analysis. However, minute intestinal flukes (MIF) such as Haplorchis taichui have eggs very similar to $O$. viverrini. Given their similar morphological appearance, it is possible that some reported $O$. viverrini eggs might belong to MIF. 
The prevalence of $S$. stercoralis and E. vermicularis in this study were underestimated due to the fact that Kato-Katz and FECT are not the appropriate diagnostic methods for these infections. The gold standard for diagnosing $S$. stercoralis is a combination of the Baermann technique and Koga-Agar cultures (Baermann, 1917; Koga et al., 1991) and for E. vermicularis, the scotch-tape anal swab technique (Cho and Kang, 1975). Hence, diversity of the helminthic infection is underestimated in our study.

Our findings on the cumulative sensitivities of Kato-Katz and FECT are in line with the observations of other authors: FECT is, in general, more sensitive than Kato-Katz for detection of LTE and the sensitivity of both techniques increases with multiple stool sampling (Glinz et al., 2010; Knopp et al., 2009, 2008).

As the applied Fasciola spp. PCR is published and tested for different areas (Alasaad et al., 2011), most likely the egg intensity was too low to be detectable or eggs were not present in the stool samples, as was observed in Vietnam (Hien et al., 2001). The use of $200 \mu \mathrm{g}$ stool for DNA extraction may also have been too small an amount to reach an acceptable level of sensitivity. For the F. buski PCR, it is possible that the published ITS2 sequence on GenBank differs from the ITS- 2 sequence of the study site, thus, the primers used were unable to detect $F$. buski eggs or the observed LTE were not caused by F. buski. Further, for both PCRs, there is the possibility of inhibition in the stool samples. 


\section{Conclusion}

It is unlikely that fascioliasis caused the observed LTE in humans. The detected prevalence of 46 LTE does not match the findings from the serum ELISA and PCR analysis. The most plausible cause is Echinostoma spp. Infections, which are reportedly endemic to Cambodia. However, risk factors for the transmission of Fasciola spp. and F. buski transmission are present. Hence, transmission may occur and public health services must remain vigilant.

\section{Acknowledgements}

We greatly appreciate the children, adolescents, parents, and cattle owners that participated in the study. We would like to thank PD Dr. med. Marcus Panning of the University of Freiburg, Germany for establishing the applied polymerase chain reactions; MD Sophanaroth Srey of CNM for actively cooperating within the cattle field survey; and Dr. Didier Ménard of the Institut Pasteur du Cambodge for providing the ELISA reader. We thank Mrs Amena Briet for her efficient English editing of the manuscript. 


\section{References}

Alasaad, S., Soriguer, R.C., Abu-Madi, M., El Behairy, A., Jowers, M.J., Baños, P.D., Píriz, A., Fickel, J., Zhu, X.-Q., 2011. A TaqMan real-time PCR-based assay for the identification of Fasciola spp. Veterinary Parasitology 179, 266-271. doi:10.1016/j.vetpar.2011.01.059

Baermann, G., 1917. Eine einfache Methode zur Auffindung von Ankylostomum (Nematoden) Larven in Erdproben. Mededeel mit H. Geneesk Laboratories Weltevreden Feestbundel, 41-47.

Chai, J.-Y., Shin, E.-H., Lee, S.-H., Rim, H.-J., 2009. Foodborne Intestinal Flukes in Southeast Asia. Korean J Parasitol 47, S69-S102. doi:10.3347/kjp.2009.47.S.S69

Chai, J.-Y., Sohn, W.-M., Na, B.-K., Van De, N., 2011. Echinostoma revolutum: Metacercariae in Filopaludina Snails from Nam Dinh Province, Vietnam, and Adults from Experimental Hamsters. The Korean Journal of Parasitology 49, 449. doi:10.3347/kjp.2011.49.4.449

Chai, J.-Y., Sohn, W.-M., Yong, T.-S., Eom, K.S., Min, D.-Y., Hoang, E.-H., Phammasack, B., Insisiengmay, B., Rim, H.-J., 2012. Echinostome Flukes Receovered from Humans in Khammouane Province, Lao PDR. The Korean Journal of Parasitology 50, 269. doi:10.3347/kjp.2012.50.3.269

Cho, S.Y., Kang, S.Y., 1975. Significance of Scotch-tape anal swab technique in diagnosis of Enterobius vermicularis infection. The Korean Journal of Parasitology 13, 102. doi:10.3347/kjp.1975.13.2.102

Glinz, D., Silué, K.D., Knopp, S., Lohourignon, L.K., Yao, K.P., Steinmann, P., Rinaldi, L., Cringoli, G., N'Goran, E.K., Utzinger, J., 2010. Comparing Diagnostic Accuracy of Kato-Katz, Koga Agar Plate, Ether-Concentration, and FLOTAC for Schistosoma mansoni and Soil-Transmitted Helminths. PLoS Negl Trop Dis 4, e754. doi:10.1371/journal.pntd.0000754

Hien, T.V., Tran, T.K., Nguye, H.C., Pham, H.D., Pham, T.H., 2001. Fascioliasis in Vietnam. Southeast Asian J. Trop. Med. Public Health 32 Suppl 2, 48-50.

Houweling, T.A., Kunst, A.E., Mackenbach, J.P., 2003. Measuring health inequality among children in developing countries: does the choice of the indicator of economic status matter? Int J Equity Health 2, 8. doi:10.1186/1475-9276-2-8

Katz, N., Chaves, A., Pellegrino, J., 1972. A simple device for quantitative stool thicksmear technique in Schistosomiasis mansoni. Rev. Inst. Med. Trop. Sao Paulo 14, 397-400.

Keiser, J., Utzinger, J., 2005. Emerging foodborne trematodiasis. Emerging Infect. Dis 11, 1507-1514.

Khieu, V., Schär, F., Marti, H., Sayasone, S., Duong, S., Muth, S., Odermatt, P., 2013. Diagnosis, Treatment and Risk Factors of Strongyloides stercoralis in Schoolchildren in Cambodia. PLoS Negl Trop Dis 7, e2035. doi:10.1371/journal.pntd.0002035

Knopp, S., Mgeni, A.F., Khamis, I.S., Steinmann, P., Stothard, J.R., Rollinson, D., Marti, H., Utzinger, J., 2008. Diagnosis of Soil-Transmitted Helminths in the Era of Preventive Chemotherapy: Effect of Multiple Stool Sampling and Use of Different Diagnostic Techniques. PLoS Negl Trop Dis 2, e331. doi:10.1371/journal.pntd.0000331 
Knopp, S., Rinaldi, L., Khamis, I.S., Stothard, J.R., Rollinson, D., Maurelli, M.P., Steinmann, P., Marti, H., Cringoli, G., Utzinger, J., 2009. A single FLOTAC is more sensitive than triplicate Kato-Katz for the diagnosis of low-intensity soiltransmitted helminth infections. Transactions of the Royal Society of Tropical Medicine and Hygiene 103, 347-354. doi:10.1016/j.trstmh.2008.11.013

Koga, K., Kasuya, S., Khamboonruang, C., Sukhavat, K., Ieda, M., Takatsuka, N., Kita, K., Ohtomo, H., 1991. A modified agar plate method for detection of Strongyloides stercoralis. Am. J. Trop. Med. Hyg. 45, 518-521.

Lee, K.-J., Bae, Y.-T., Kim, D.-H., Deung, Y.-K., Ryang, Y.-S., Kim, H.-J., Im, K.-I., Yong, T.-S., 2002. Status of intestinal parasites infection among primary school children in Kampongcham, Cambodia. Korean J Parasitol 40, 153-155. doi:10.3347/kjp.2002.40.3.153

Marti, H., Escher, E., 1990. [SAF--an alternative fixation solution for parasitological stool specimens]. Schweiz Med Wochenschr 120, 1473-1476.

Marti, H., Koella, J.C., 1993. Multiple stool examinations for ova and parasites and rate of false-negative results. J. Clin. Microbiol. 31, 3044-3045.

McKenzie, F.E., 2005. Polyparasitism. Int. J. Epidemiol. 34, 221-222. doi:10.1093/ije/dyh399

Park, S.K., Kim, D.-H., Deung, Y.-K., Kim, H.-J., Yang, E.-J., Lim, S.-J., Ryang, Y.-S., Jin, D., Lee, K.-J., 2004. Status of intestinal parasite infections among children in Bat Dambang, Cambodia. Korean J Parasitol 42, 201-203. doi:10.3347/kjp.2004.42.4.201

Petney, T.N., Andrews, R.H., 1998. Multiparasite communities in animals and humans: frequency, structure and pathogenic significance. International Journal for Parasitology 28, 377-393. doi:10.1016/S0020-7519(97)00189-6

Quang, T.D., Duong, T.H., Richard-Lenoble, D., Odermatt, P., Khammanivong, K., 2008. [Emergence in humans of fascioliasis (from Fasciola gigantica) and intestinal distomatosis (from Fasciolopsis buski) in Laos]. Sante 18, 119-124. doi:10.1684/san.2008.0110

Radomyos, B., Wongsaroj, T., Wilairatana, P., Radomyos, P., Praevanich, R., Meesomboon, V., Jongsuksuntikul, P., 1998. Opisthorchiasis and intestinal fluke infections in northern Thailand. Southeast Asian J. Trop. Med. Public Health 29, 123-127.

Sayasone, S., Mak, T.K., Vanmany, M., Rasphone, O., Vounatsou, P., Utzinger, J., Akkhavong, K., Odermatt, P., 2011. Helminth and Intestinal Protozoa Infections, Multiparasitism and Risk Factors in Champasack Province, Lao People's Democratic Republic. PLoS Negl Trop Dis 5, e1037. doi:10.1371/journal.pntd.0001037

Sayasone, S., Vonghajack, Y., Vanmany, M., Rasphone, O., Tesana, S., Utzinger, J., Akkhavong, K., Odermatt, P., 2009. Diversity of human intestinal helminthiasis in Lao PDR. Transactions of the Royal Society of Tropical Medicine and Hygiene 103, 247-254. doi:10.1016/j.trstmh.2008.10.011

Sinuon, M., Anantaphruti, M.T., Socheat, D., 2003. Intestinal helminthic infections in schoolchildren in Cambodia. Southeast Asian J. Trop. Med. Public Health 34, 254-258. 
Sohn, W.-M., Chai, J.-Y., Na, B.-K., Yong, T.-S., Eom, K.S., Park, H., Min, D.-Y., Rim, H.-J., 2013. Echinostoma macrorchis in Lao PDR: Metacercariae in Cipangopaludina Snails and Adults from Experimentally Infected Animals. The Korean Journal of Parasitology 51, 191. doi:10.3347/kjp.2013.51.2.191

Sohn, W.-M., Chai, J.-Y., Yong, T.-S., Eom, K.S., Yoon, C.-H., Sinuon, M., Socheat, D., Lee, S.-H., 2011a. Echinostoma revolutum Infection in Children, Pursat Province, Cambodia. Emerg Infect Dis 17, 117-119. doi:10.3201/eid1701.100920

Sohn, W.-M., Kim, H.-J., Yong, T.-S., Eom, K.S., Jeong, H.-G., Kim, J.-K., Kang, A.-R., Kim, M.-R., Park, J.-M., Ji, S.-H., Sinuon, M., Socheat, D., Chai, J.-Y., 2011 b. Echinostoma ilocanum infection in Oddar Meanchey Province, Cambodia. Korean J. Parasitol. 49, 187-190. doi:10.3347/kjp.2011.49.2.187

Steinmann, P., Utzinger, J., Du, Z.-W., Zhou, X.-N., 2010. Chapter 2 - Multiparasitism: A Neglected Reality on Global, Regional and Local Scale, in: Important Helminth Infections in Southeast Asia: Diversity and Potential for Control and Elimination, Part B. Academic Press, pp. 21-50.

Tum, S., Puotinen, M.L., Copeman, D.B., 2004. A geographic information systems model for mapping risk of fasciolosis in cattle and buffaloes in Cambodia. Vet. Parasitol 122, 141-149. doi:10.1016/j.vetpar.2004.03.016

Tum, S., Puotinen, M.L., Skerratt, L.F., Chan, B., Sothoeun, S., 2007. Validation of a geographic information system model for mapping the risk of fasciolosis in cattle and buffaloes in Cambodia. Vet. Parasitol 143, 364-367. doi:10.1016/j.vetpar.2006.08.033

Tungtrongchitr, A., Chiworaporn, C., Praewanich, R., Radomyos, P., Boitano, J.J., 2007. The potential usefulness of the modified Kato thick smear technique in the detection of intestinal sarcocystosis during field surveys. Southeast Asian J. Trop. Med. Public Health 38, 232-238.

Valero, M.A., Perez-Crespo, I., Periago, M.V., Khoubbane, M., Mas-Coma, S., 2009. Fluke egg characteristics for the diagnosis of human and animal fascioliasis by Fasciola hepatica and F. gigantica. Acta Tropica 111, 150-159. doi:10.1016/j.actatropica.2009.04.005

Waree, P., Polseela, P., Pannarunothai, S., Pipitgool, V., 2001. The present situation of paragonimiasis in endemic area in Phitsanulok Province. Southeast Asian J. Trop. Med. Public Health 32 Suppl 2, 51-54. 


\section{Figure legends}

Figure 1: Flow chart of cross-sectional study on helminthic infections in Cambodian schoolchildren

Figure 2: Cumulative prevalence of combined Kato-Katz and FECT results for the number of examined stool samples

Figure 3: Large trematode egg in Kato-Katz fecal smear of Damrei Chhlang (Magnification 400x) 
Table 1: Prevalence of intestinal parasitic infections in schoolchildren diagnosed by Kato-Katz and FECT

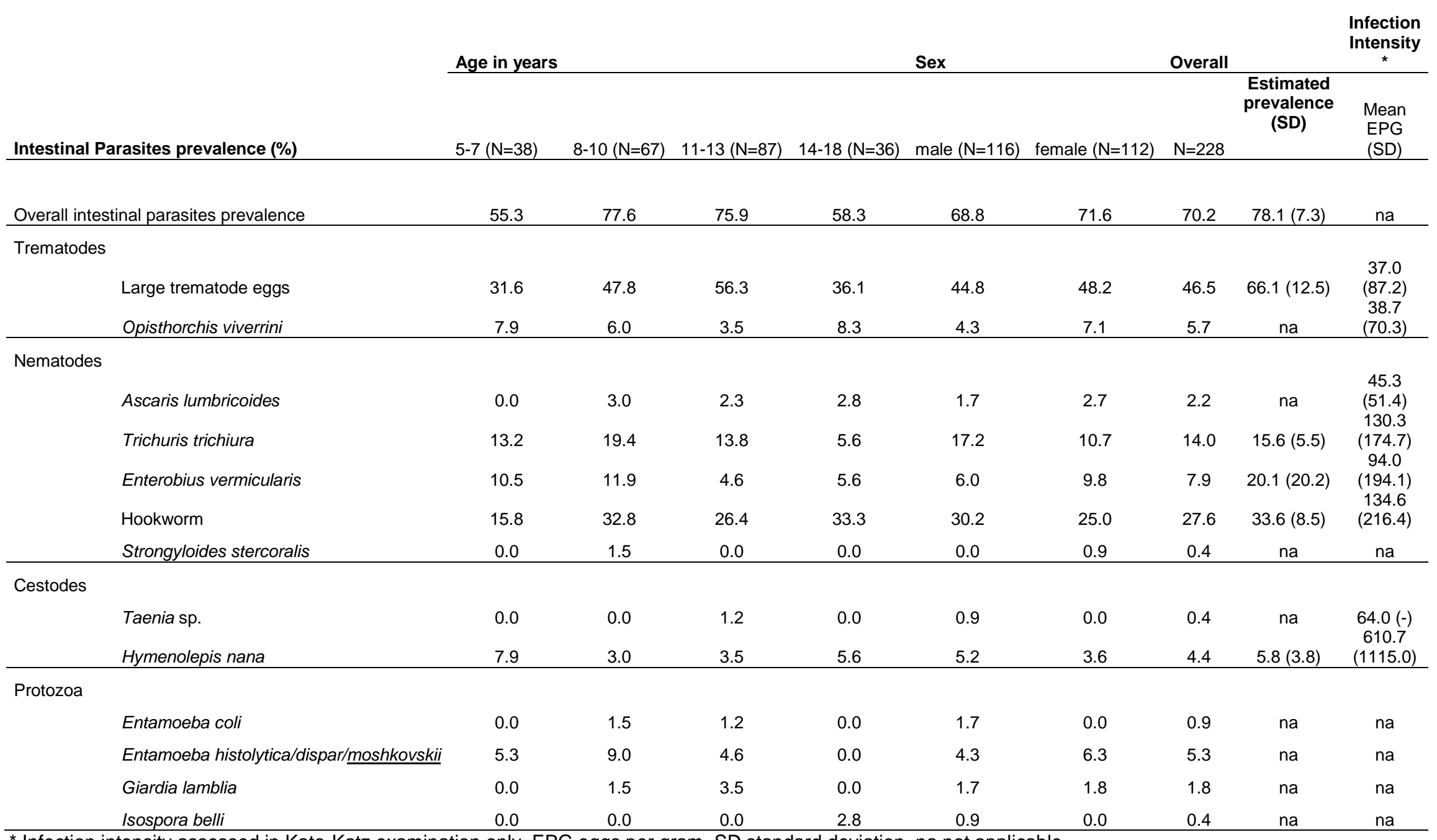

* Infection intensity assessed in Kato-Katz examination only, EPG eggs per gram, SD standard deviation, na not applicable 
Table 2: Association of risk factors with infection with large trematode eggs (univariable regression analysis, $\mathrm{n}=228$ )

\begin{tabular}{lcccc}
\hline Risk factors & $\begin{array}{c}\% \text { of } \\
\text { infected }\end{array}$ & $\begin{array}{c}\% \text { of non- } \\
\text { infected }\end{array}$ & OR 95\% CI P-value \\
\hline
\end{tabular}

\section{Demographics}

Socio-economic status (versus most poor)

$\begin{array}{llllll}\text { poor } & 32.1 & 34.4 & 1.1 & 0.6-2.0 & 0.870 \\ \text { least poor } & 36.8 & 30.3 & 1.4 & 0.7-2.6 & 0.330\end{array}$

Age (versus 5 to 7 years)

8 to 10 years

11 to 13 years

$\begin{array}{lllll}30.2 & 28.7 & 2.0 & 0.9-4.6 & 0.109\end{array}$

14 to 18 years

46.2

$31.2 \quad 2.8 \quad 1.2-6.2 \quad 0.012$

12.3

$18.9 \quad 1.2 \quad 0.5-3.2 \quad 0.681$

Sex (female versus male)

50.9

47.5

$1.1 \quad 0.7-1.9$

0.608

Work in a rice field within the last year (yes versus no)

59.4

54.1

$1.2 \quad 0.7-2.1$

0.418

Help for water collection (yes versus no)

65.

67.2

$0.9 \quad 0.5-1.6$

0.736

\section{Eating habits}

Aquatic plants (yes versus no)

Raw aquatic plants (yes versus no)*

Pork meat (yes versus no)

Raw pork meat (yes versus no)

Prawns (yes versus no)

Raw prawns (yes versus no)

Chicken/Duck (yes versus no)

Fish dishes (yes versus no)

Fermented fish sauce (yes versus no)*

Raw fermented fish sauce (yes versus no)

Crab (yes versus no)

$\begin{array}{ccccc}81.1 & 75.2 & 1.5 & 0.8-2.8 & 0.238 \\ 17.0 & 7.4 & 2.6 & 1.1-6.0 & 0.029 \\ 85.9 & 91.0 & 0.6 & 0.3-1.4 & 0.227 \\ 0.9 & 0.8 & 1.2 & 0.1-18.7 & 0.920 \\ 34.0 & 27.1 & 1.4 & 0.8-2.4 & 0.258 \\ 1.9 & 0.8 & 2.3 & 0.2-26.0 & 0.493 \\ 72.6 & 67.2 & 1.3 & 0.7-2.3 & 0.374 \\ 99.1 & 97.5 & 2.6 & 0.3-25.8 & 0.402 \\ 86.8 & 75.4 & 2.1 & 1.1-4.3 & 0.032 \\ 6.6 & 11.5 & 0.5 & 0.2-1.4 & 0.210 \\ 33.0 & 35.3 & 0.9 & 0.5-1.6 & 0.724 \\ 11.3 & 13.9 & 0.8 & 0.4-1.7 & 0.555 \\ 38.7 & 38.5 & 1.0 & 0.6-1.7 & 0.981 \\ 1.9 & 0.8 & 2.3 & 0.2-26.0 & 0.493\end{array}$

Raw crabs (yes versus no)

Cattle meat dishes (yes versus no)

Raw cattle meat dishes (yes versus no) 


\section{Personal hygiene habits}

Usual places of defecation

Toilet (yes versus no)

Behind the house (yes versus no)

$\begin{array}{lllll}75.5 & 76.2 & 1.0 & 0.5-1.8 & 0.894\end{array}$

Forest (yes versus no)

$\begin{array}{lllll}17.9 & 18.9 & 0.9 & 0.5-1.8 & 0.857\end{array}$

Into the water (yes versus no)

$\begin{array}{lllll}5.7 & 5.7 & 1.0 & 0.3-3.0 & 0.980\end{array}$

$\begin{array}{lllll}0.9 & 0.8 & 1.2 & 0.1-18.7 & 0.920\end{array}$

Hand washing before eating the last time (yes versus no)

$\begin{array}{lllll}78.3 & 82.0 & 0.8 & 0.4-1.5 & 0.488\end{array}$

Hand washing before eating the last time (yes versus no)

$\begin{array}{lllll}64.2 & 62.3 & 1.1 & 0.6-1.9 & 0.772\end{array}$

Using soap for hand washing (yes versus no)

37.7

$\begin{array}{llll}43.4 & 0.8 & 0.5-1.3 & 0.382\end{array}$

Daily bathing in river/pond (yes versus no)*

49.1

$\begin{array}{llll}40.2 & 1.4 & 0.8-2.4 & 0.178\end{array}$

*included in the multivariable logistic regression 